\title{
Spectral interferometric technique to measure ellipsometric phase of a thin-film structure
}

\author{
Petr Hlubina, ${ }^{*}$ Dalibor Ciprian, Jiri Lunacek \\ Department of Physics, Technical University Ostrava, \\ 17. listopadu 15, 70833 Ostrava-Poruba, Czech Republic \\ *Corresponding author: petr.hlubina@vsb.cz
}

\begin{abstract}
Compiled August 8, 2009
A two-step white-light spectral interferometric technique is used to retrieve the ellipsometric phase of a thin-film structure from the spectral interferograms recorded in a polarimetry configuration with a birefringent crystal. In the first step, the phase difference between $p$ - and $s$-polarized waves propagating in the crystal alone is retrieved. In the second step, the additional phase change that the polarized waves undergo on reflection from the thin-film structure is retrieved. The new method is used in determining the thin-film thickness from ellipsometric phase measured for $\mathrm{SiO}_{2}$ thin film on a $\mathrm{Si}$ substrate in a range from 550 to 900 $\mathrm{nm}$. The thicknesses of three different samples obtained are compared with those resulting from polarimetric measurements and good agreement is confirmed. (C) 2009 Optical Society of America

OCIS codes: $120.3180,120.5050,120.5410,120.5700,240.0310$.
\end{abstract}

Ellipsometry and interferometry are widely used techniques for the analysis of surfaces and thin films. Various ellipsometry types are employed to determine the thickness and the optical properties of thin film. Ellipsometric measurements performed at a single wavelength and a fixed angle of incidence are used to determine the amplitude and phase changes of $p$ - and $s$ polarized components to provide the film thickness and optical constants [1]. Measurements by spectroscopic ellipsometry provide the results over a wide wavelength range with greater precision and accuracy [2]. The use of white-light interferometry [3] was extended into the spectral domain $[4,5]$ where the phase of the reflected wave, which changes as a function of wavelength and layer thickness $[6,7]$, is inscribed in the recorded interferogram (channeled spectrum). There are also available single-wavelength interferometric ellipsometers [8] that employ Michelson or Mach-Zehnder interferometer. In this Letter, a two-step white-light spectral interferometric technique for measuring ellipsometric phase of a thinfilm sample is presented. The technique, which is a modification of the technique originally employed for surface plasmon resonance sensing [9], utilizes a polarimetry configuration with a birefringent crystal that allows to obtain the channeled spectrum. Two such spectra, one including reflection of $p$ - and $s$-polarized waves from the thin-film sample and the other one without the reflection, are used to retrieve the ellipsometric phase. Moreover, in the same polarimetric configuration, the ratio between the reflectances of both polarization states is measured.

Let us consider a simple experimental setup as shown in Fig. 1 proposed for measuring the wavelength dependence of ellipsometric phase of a thin-film structure. The collimated beam from a white-light source passes through a polarizer oriented $45^{\circ}$ with respect to the plane of incidence so that both $p$ - and $s$-polarized compo- nents are generated. Next, the beam passes through the birefringent crystal whose optical axis is perpendicular to the plane of incidence and the phase delays $\phi_{p, s}(\lambda)$ for the $p$ - and $s$-polarized components are introduced. Finally, these retarded components undergo due to reflection from a thin-film structure at the fixed angle $\alpha$ of the incidence the amplitude and phase changes that are related to the complex reflection coefficients [1]

$$
r_{p, s}(\lambda)=\sqrt{R_{p, s}(\lambda)} \exp \left[\mathrm{i} \delta_{p, s}(\lambda)\right],
$$

where $R_{p, s}(\lambda)$ and $\delta_{p, s}(\lambda)$ are the wavelength-dependent reflectances and phase changes on reflection for both polarizations, respectively. The two orthogonally polarized components are mixed with an analyzer oriented $45^{\circ}$ with respect to the plane of incidence and their interference is resolved by a spectrometer as a channeled spectrum. The information provided by standard reflection ellipsometry is the ellipsometric phase $\Delta(\lambda)=$ $\delta_{p}(\lambda)-\delta_{s}(\lambda)$ and the reflection ratio $\sqrt{R_{p}(\lambda)} / \sqrt{R_{s}(\lambda)}=$ $\tan \Psi(\lambda)$ of a thin-film structure for $p$ - and $s$-polarized components, where $\Psi(\lambda)$ is the ellipsometric angle.

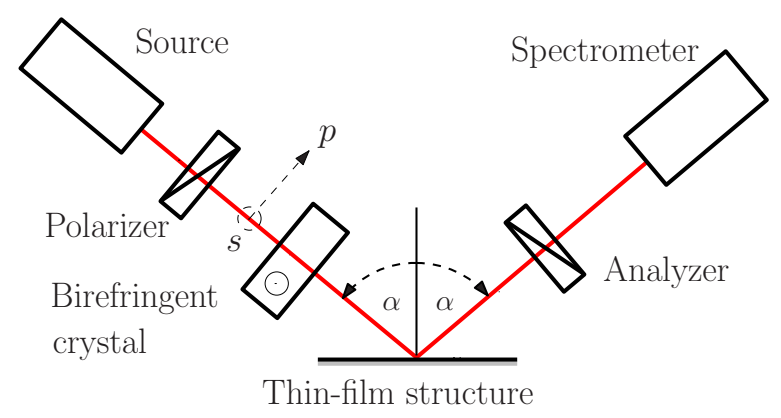

Fig. 1. (Color online) Experimental setup with a polarimetry configuration and a birefringent crystal.

In order to retrieve the wavelength-dependent ellipso- 
metric phase $\Delta(\lambda)$ from the recorded channeled spectra, the measurement procedure is made in two steps. In the first one, the phase difference $\phi_{B C}(\lambda)=\phi_{p}(\lambda)-\phi_{s}(\lambda)$ between the $p$ - and $s$-polarized components (retardance) introduced by the birefringent crystal of a suitable thickness $t$ is retrieved from the spectral interferogram. It is obtained in the setup shown in Fig. 1 when the thinfilm structure is removed and the angle of the incidence $\alpha$ is adjusted to be $90^{\circ}$. The spectrum recorded by the spectrometer of a Gaussian response function can be represented in the form [10]

$$
I(\lambda)=I_{0}(\lambda)\left\{1+V_{\mathrm{R}}(\lambda) \cos \left[\phi_{B C}(\lambda)\right]\right\},
$$

where $I_{0}(\lambda)$ is the source spectrum and $V_{\mathrm{R}}(\lambda)=$ $\exp \left\{-\left(\pi^{2} / 2\right)\left[G_{\mathrm{f}}(\lambda) t \Delta \lambda_{\mathrm{R}} / \lambda^{2}\right]^{2}\right\}$ is a visibility term dependent on both the group birefringence $G_{\mathrm{f}}(\lambda)$ of the crystal and width $\Delta \lambda_{\mathrm{R}}$ of the spectrometer response function. In the second step, the spectral interferogram is recorded in the setup shown in Fig. 1 for the fixed angle $\alpha$ of the incidence. The spectrum recorded by the spectrometer is given by

$$
I^{\prime}(\lambda)=I^{\prime}{ }_{0}(\lambda)\left\{1+V_{\mathrm{R}}(\lambda) V(\lambda) \cos \left[\phi_{B C}(\lambda)+\Delta(\lambda)\right]\right\},
$$

where $I_{0}^{\prime}{ }_{0}(\lambda)$ is the reference spectrum and $V(\lambda)=$ $2 \sqrt{R_{p}(\lambda) R_{s}(\lambda)} /\left[R_{p}(\lambda)+R_{s}(\lambda)\right]$ is a visibility term due to the thin-film structure. From the spectral interferogram recorded in the second step, the overall phase $\Phi(\lambda)$ is retrieved giving the ellipsometric phase $\Delta(\lambda)$ of the structure as the difference between the overall phase $\Phi(\lambda)$ and the retardance $\phi_{B C}(\lambda)$ of the birefringent crystal.

The procedure for the measurement of the reflectance ratio $R_{p}(\lambda) / R_{s}(\lambda)$ or $\tan ^{2} \Psi(\lambda)$ consists of three steps: first, with the source blocked, the background spectrum $I_{b k g}(\lambda)$ is measured; second, with the analyzer oriented perpendicularly to the plane of the incidence, the reflection spectrum $I_{s}(\lambda)$ for $s$ polarization is measured; and third, with the analyzer oriented parallel to the plane of the incidence, the reflection spectrum $I_{p}(\lambda)$ for $p$ polarization is measured. The reflectance ratio $R_{p}(\lambda) / R_{s}(\lambda)$ of the structure is given by

$$
R_{p}(\lambda) / R_{s}(\lambda)=\left[I_{p}(\lambda)-I_{b k g}(\lambda)\right] /\left[I_{s}(\lambda)-I_{b k g}(\lambda)\right] .
$$

The experimental setup used for measurement of ellipsometric phase $\Delta(\lambda)$ of a thin-film structure is shown schematically in Fig. 1 and it consists of a white-light source: a halogen lamp HL-2000 (Ocean Optics) with launching optics, an optical fiber and a collimating lens, a crystal of birefringent quartz of thickness $t=6 \mathrm{~mm}$, Glan Taylor calcite polarizer and analyzer (Thorlabs), a thin-film structure, a microscope objective, micropositioners, a read optical fiber, a fiber-optic spectrometer S2000 (Ocean Optics), an A/D converter and a personal computer. The spectrometer has a spectral operation range from 350 to $1000 \mathrm{~nm}$ and its spectral resolution is limited by a Gaussian response function with the width $\Delta \lambda_{\mathrm{R}}=2.7 \mathrm{~nm}$.

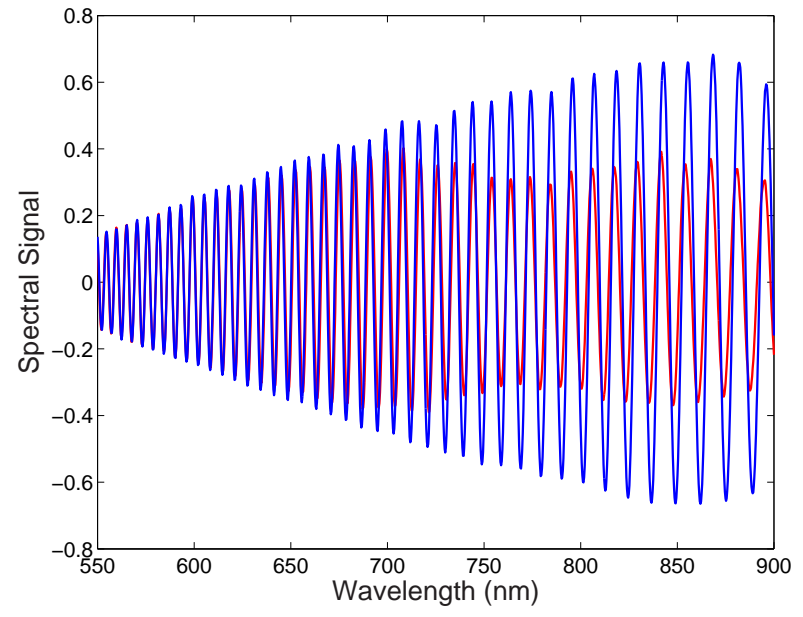

Fig. 2. (Color online) Measured spectral signal for a birefringent quartz crystal and its change due to the third sample of a thin-film structure $\left(\alpha \approx 45^{\circ}\right)$.

We demonstrate the ability of our new method in measuring ellipsometric phase $\Delta(\lambda)$ for three different samples of $\mathrm{SiO}_{2}$ thin film on a $\mathrm{Si}$ substrate. The $\mathrm{SiO}_{2}$ thin films on the Si wafer were prepared by using a dry oxidation process when the wafers were annealed in a furnace at $1200^{\circ} \mathrm{C}$. Three annealing times were selected in order to prepare $\mathrm{SiO}_{2}$ thin film with three different thicknesses ranging approximately from 300 to $450 \mathrm{~nm}$. Procedure used to retrieve a relative spectral phase $\Phi(\lambda)$ from the recorded spectral interferogram comprises two steps. In the first step, the reference spectrum $I_{0}(\lambda)$ is retrieved from the channeled spectrum $I(\lambda)$. It is obtained as the inverse Fourier transform of the zeroorder component of the Fourier spectrum of the recorded channeled spectrum. In the second step, the spectral phase function $\Phi(\lambda)$ is retrieved from the spectral signal $S(\lambda)=I(\lambda) / I_{0}(\lambda)-1$ using a procedure based on a windowed Fourier transform [11]. As an example, Fig. 2 shows in blue the measured spectral signal in which only the effect of the birefringent crystal is inscribed. It is clearly seen that a large number of the spectral interference fringes of sufficiently high visibility are resolved. The number of the spectral fringes in the wavelength range from 550 to $900 \mathrm{~nm}$ is given by the group path difference $G_{\mathrm{f}}(\lambda) t$ of the birefringent quartz crystal [10].

Similarly, Fig. 2 shows in red the measured spectral signal for the third sample of the thin-film structure and the angle $\alpha$ of the incidence of approximately $45^{\circ}$. It can be noted from Fig. 2 that the thin-film structure affects both the visibility and phase of the spectral interference fringes. The spectral fringes in red are in comparison with those in blue shifted to shorter wavelengths in a wavelength range from 764 to $900 \mathrm{~nm}$. That means that the ellipsometric phase $\Delta(\lambda)$ is in this wavelength range negative. This fact is illustrated by curve 3 in Fig. 3 which shows the retrieved ellipsometric phase $\Delta(\lambda)$ as a function of wavelength for all samples. The ellipsometric 
phase for the third sample has two apparent extrema, while the one for the first sample has no extrema. In the same figure are also shown the theoretical curves obtained by a least-square algorithm [11]. Theoretical curves are computed by using well-known relations for the complex reflection coefficients of $p$ - and $s$-polarized waves reflected from a non-absorbing thin film on an absorbing substrate [1]. Using the optical parameters of both $\mathrm{SiO}_{2}$ and $\mathrm{Si}[5,12]$, the thicknesses of the $\mathrm{SiO}_{2}$ thin film for three measured samples are determined as 286.1, 333.6 and $445.7 \mathrm{~nm}$, respectively. These values are in good agreement with those obtained by spectral interferometry and normal incidence reflectometry [13].

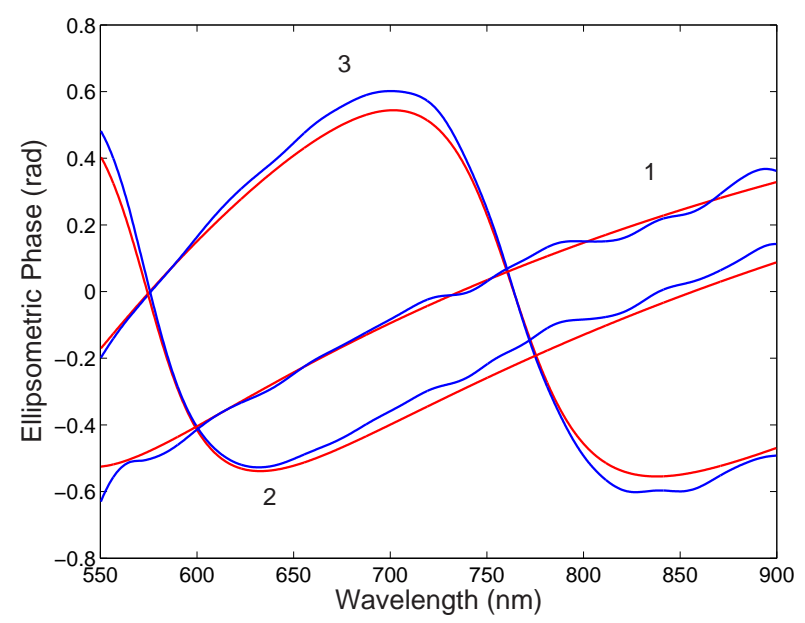

Fig. 3. (Color online) Measured ellipsometric phase $\Delta(\lambda)$ as a function of wavelength for three different samples $\left(\alpha \approx 45^{\circ}\right)$. The curves without ripples - theory.

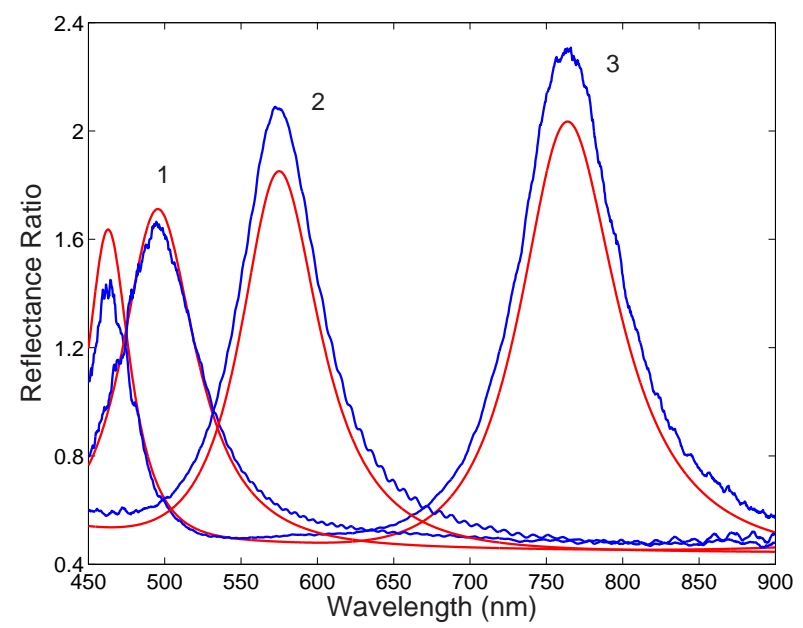

Fig. 4. (Color online) Measured and theoretical reflectance ratio $R_{p}(\lambda) / R_{s}(\lambda)$ as a function of wavelength for three different samples $\left(\alpha \approx 45^{\circ}\right)$.

Finally, the reflectance ratios $R_{p}(\lambda) / R_{s}(\lambda)$ measured as a function of wavelength for all the three samples are shown in Fig. 4 together with the theoretical dependencies. The right orientations of the analyzer in measuring the reflectances $R_{p}(\lambda)$ and $R_{s}(\lambda)$ are attained by disappearance of the spectral interference fringes. The ratio $R_{p}(\lambda) / R_{s}(\lambda)$ measured for the third sample includes two maxima, whereas the remaining curves include only one maximum. Positions of the maxima in the measured curves agree well with theory. Apparent is the difference in absolute values which is probably caused by nonideal alignment of the analyzer with respect to the detection part. The thicknesses of the $\mathrm{SiO}_{2}$ thin film determined from the measured reflectance ratios for the samples are as follows $286.5,333.9$ and $445.7 \mathrm{~nm}$. These values are in very good agreement with those obtained from the ellipsometric phase measurements.

In conclusion, a simple two-step white-light spectral interferometric technique for measuring the ellipsometric phase of a thin-film structure has been presented. The technique utilizes a cost-effective instrumentation of a polarimetry configuration with a birefringent crystal and a fiber-optic spectrometer. The spectrometer records two channeled spectra whose processing by a windowed Fourier transform gives the ellipsometric phase. The feasibility of the technique has been demonstrated in measuring the thickness of $\mathrm{SiO}_{2}$ thin film on a $\mathrm{Si}$ substrate. We confirmed that the thickness agree well with that measured in the same setup by polarimetry. The use of the technique can be extended, for example, for measuring the ellipsometric phase of complex structures at various angles of incidence or the phase changes due to both surface plasmon resonances and low-birefringent fibers.

The research has been partially supported by the Ministry of Education, Youth and Sports of the Czech Republic through grant MSM6198910016.

\section{References}

1. R. M. A. Azzam and N. M. Bashara, Ellipsometry and Polarized Light (North-Holland, 1977).

2. G. E. Jellison, Jr., Thin Solid Films 290-291, 40 (1996).

3. S. W. Kim, G. H. Kim, Appl. Opt. 38, 5968 (1999).

4. U. Schnell, R. Dändliker, S. Gray, Opt. Lett. 21, 528 (1996)

5. P. Hlubina, D. Ciprian, J. Lunacek, and M. Lesnak, Opt. Express 14, 7678 (2006).

6. T. Doi, K. Toyoda, and Y. Tanimura, Appl. Opt. 36, 7157 (1997).

7. K. M. Medicus, M. Chaney, J. E. Brodziak, Jr., and A. Davies, Appl. Opt. 46, 2027 (2007).

8. L. R. Watkins, Appl. Opt. 47, 2998 (2008).

9. Z. Zheng, Y. Wan, X. Zhao, and J. Zhu, Appl. Opt. 48, 2491 (2009).

10. P. Hlubina, D. Ciprian, and L. Knyblova, Opt. Commun. 260, 535 (2006).

11. P. Hlubina, J. Lunacek, D. Ciprian, and R. Chlebus, Opt. Commun. 281, 2349 (2008).

12. E. Palik, Handbook of Optical Constants of Solids (Academic, San Diego, Calif., 1985), Vol. I.

13. P. Hlubina, J. Lunacek, D. Ciprian, and R. Chlebus, Appl. Phys. B 92, 203 (2008). 